\title{
La comida en común espartana como mecanismo de diferenciación e integración social
}

\author{
Juan Miguel Casillas y César Fornis*
}

Como inicio del presente artículo conviene precisar los diversos términos griegos utilizados por las fuentes clásicas para designar los banquetes propios de la cultura doria y, más concretamente, los celebrados en Esparta y Creta. Las denominaciones habituales que podemos encontrar son las de $\sigma 0 \sigma \sigma \tau i \alpha$ y $\phi i \delta i \tau i \alpha$ ', ambas utilizadas para designar la «comida en común» espartana, a las que debemos añadir las variantes

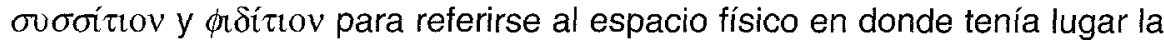
reunión de los comensales. El vocablo $\phi i \delta i \tau i \alpha$, si optamos por la opinión expresada por Aristóteles ${ }^{2}$, sería una acepción posterior que fue adoptada por Esparta del más antiguo término de $\alpha^{\prime} \delta \rho p \varepsilon \hat{\alpha} \alpha$, procedente de Creta, isla con la que Lacedemonia compartía múltiples similitudes que la tradición atribuyó al único y legendario autor de la Retra ${ }^{3}$. No obstante, es Plutarco quien nos remite al sentido originario de $\phi \imath \delta \imath \tau i \alpha$ al relacionarlo

\section{* Universidad Complutense.}

Este artículo tiene como base un trabajo en inglés presentado a la revista Papers from the Institute of Archaeology, bajo el título «An Appreciation on the Social Function of the Spartan $\sigma v \sigma-$

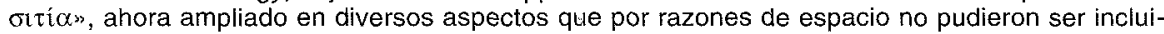
dos entonces. Agradecemos al profesor Domingo Plácido sus inestimables consejos en la elaboración definitiva del artículo, aunque somos exclusivamente responsables de cuantos errores pudiera contener el mismo. Asimismo, agradecemos a la Oficinal Municipal de Fomento a la Investigación de la Comunidad de Madrid y al Profesor Julio Mangas, en representación de la Universidad Complutense, la posibilidad de haber contado con una ayuda económica que ha permitido nuestra estancia en el Institute of Classical Studies de la Universidad de Londres.

Otras variantes menos habituales son: $\phi \imath \lambda i \tau l \alpha$ (Plu. Phoc. 20; Cic. Tusc. 5.34.98), $\phi \varepsilon \imath \delta \tau i \alpha$ (X. HG. 5.4.28), $\phi \varepsilon ı$ íiıov (IG. V.1 1507) y $\phi \varepsilon i \delta \varepsilon i \tau$ ov (IG. V.1 128.13).

2 Arist. Pol. 1272 a 5.

3 En efecto, Licurgo viajó en diversas ocasiones a Creta, antes y después de su partida definitiva de Esparta. Incluso tras producirse su muerte, algunas localidades cretenses se atribuyeron el prestigio de ser el lugar donde falleció el mítico personaje o donde se situaba su tumba (cfr. Plu.

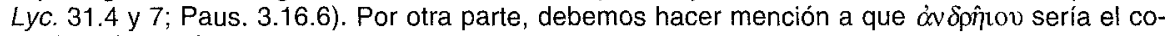
medor público de los cretenses. 
con el término $\phi \lambda \hat{\lambda} i \alpha$, la amistad o afecto que presidía estas comidas en común, pero no descarta la posibilidad de que proceda de ćsítı $\alpha$, es decir, el acto de comer ${ }^{4}$. Una última pero indispensable referencia ha de hacerse a Jenofonte, buen conocedor de las costumbres lacedemonias, quien en sus escritos utiliza los términos $\sigma v \sigma \kappa \eta v i ́ \alpha$ o $\sigma v \sigma \kappa \eta ́ v ı l \alpha$ para aludir a la vida en común y a los banquetes militares desarrollados en Esparta, palabra que no encontramos en ningún otro autor clásico ${ }^{5}$.

Precisada la terminología, nos parece oportuno recordar que en los últimos años el tema del sumposion griego en general y la sussitia en particular ha despertado un creciente interés que se ha concretado en la publicación de diversos trabajos, entre los que destacamos el de T. J. Figueira, centrado en la vertiente económica, M. Nafissi, desde un enfoque situado en el plano ideológico y N. R. E. Fisher, sociológico en esencia, pero con implicaciones políticas ${ }^{6}$. La importancia de esta institución espartana es tal que se extendía a todos los ámbitos de la vida lacedemonia, dejando sentir su impronta en los planos político, económico, militar, educativo, moral y sexual. Es nuestra intención analizar aqui el sistema de integración y diferenciación social que supuso la participación en estos banquetes en el marco de las costumbres espartanas a través, principalmente, de las referencias aportadas por los autores antiguos.

Podriamos definir la $\sigma v \sigma \sigma \tau i \alpha$ como la reunión de los varones adultos espartiatas de pleno derecho (ó $\mu \mathrm{olol}$ ) con la finalidad de estrechar y reforzar los vínculos de unión que hacían posible su predominio sociopolítico mediante el acto simbólico de una comida en común ${ }^{7}$. Así, en estos

Plu. Lyc. 12.1-2.

X. LaC. 5.2 .

T. J. FIGUEIRA, «Mess Contributions and Subsistence at Sparta», TAPhA 114 (1984), 87109; M. NAFISSI, La nascità del "Kosmos». Studi sulla storia e la società di Sparta, Perugia, 1991; N.R.E. FISHER, "Dink, Hybris and the Promotion of Harmony in Sparta", en A. POWELL (ed.), Classical Sparta: Techniques behind her Success, Londres, 1989, 26-50. Cfr. también, O. MurraY, "The Greek Symposion in History", en Tria Corda: Scritti in onore di A. Momigliano, Como, 1983, 266-70; E. DAvID, "The Spartan Syssitia and Plato's Law”, AJPh 99 (1978), 486-95 que analiza, con carácter general, las controversias planteadas por esta institución en las Leyes de Platón; K.T.M. Chrimes, Ancient Sparta, Manchester, 1949, 218, 230 y 245; H. Michel, Sparta, Cambridge, 1964, 39, 236, 295 y 331; A.H.M. JONES, Sparta, Oxford, 1967, 36-7 y 153; P.A. CARTLEDgE, Sparta and Lakonia. A Regional History, 1300-362 B.C., Londres, 1979, 170-1 y 174; J.T. HookER, The Ancient Spartans, Londres, 1980, 116-8 y W.G. ForRest, A History of Sparta, 930-192 B.C., Londres, $1980^{2}, 45$ y 52.

La sussitia era una de las bases más destacadas del sistema espartano que iba contra la tradición del oikos: los valores familiares se ven combatidos y finalmente aplastados, mientras la única función del estado es la de producir ciudadanos vigorosos que se conviertan en buenos soldados y en esto juega un papel destacado la comida en común. Cfr. M. Austin y P. VIDALNAQUET, EConomia y Sociedad en la Antigua Grecia (trad. de T. de Lozoya), Barcelona, 1986, 85. 
banquetes se daban cita prácticamente aquellos que participaban en la Ekklesia espartana -más los efebos, en el estadio final de la agoge- y que, por consiguiente, tomaban las decisiones políticas. Por tanto, podemos considerar que en las sussitiai se fraguaban y discutían desde diferentes puntos de vista temas que ulteriormente eran encauzados de manera oficial a través de la Asamblea ${ }^{8}$. Haciendo buenas las palabras de B. L. Kunstler, «agelai y sussitiai funcionaban a modo de minipoleis dentro del más amplio organigrama del estado espartano" ${ }^{9}$. La enorme significación y trascendencia de esta práctica social es aún mayor si tenemos presente la endémica oligandria padecida por Esparta, basada en un restringido acceso a la ciudadanía y en el alto porcentaje de población sometida ${ }^{10}$. En este sentido, hemos de concebir la sussitia como una especie de logia - si bien, lejos de ser ajena, inserta en las coordenadas políticas estatales - que hermanaba e identificaba a sus integrantes en unos mismos intereses y objetivos e incluso requería la aceptación de los nuevos miembros por parte de los antiguos y el secreto acerca de las conversaciones y actos desarrollados durante las sesiones ${ }^{11}$. Este tipo

8 Y no exclusivamente el recuerdo de «bellas» acciones realizadas por los ciudadanos que menciona X. Lac. 5.6. Así, tenernos el ejemplo de Esfodrias que, a punto de ser condenado a muerte por los éforos, fue apoyado por sus compañeros de mesa, entre los que se encontraba Arquidamo, hijo del rey Agesilao Il y hetairos de Cleónimo, hijo a su vez de Esfodrias (X. HG. 5.4.25-28).

9 B. L. KUnSTLER, Women and the Development of the Spartan Polis: Study of Sex Roles in Classical Antiquity, diss. St. Univ. of New York, Nueva York, 1983, 448. Las agelai, literalmente "rebaños para ser guiados", eran grupos en los que los jóvenes espartiatas desarrollaban su instrucción militar bajo la atenta mirada de sus amantes adultos; cfr. P. CHARTRAINE, Études sur le vocabulaire grecque, París, 1956, 32-3 y P. VIDAL-NAQUET, «Greek Adolescence", en The Black Hunter (trad. al inglés de A. Szegedy-Maszad), Baltimore-Londres, 1986, 142-56. M. NAFISSI, op. cit., 177 pone en conexión las agelai y las sussitiai, aunque admite que el principal mecanismo de regulación de este «rite de passage» del adolescente no es conocido en profundidad.

to Para la población espartiata, véase Hdt. 7.234.2, 9.10.1, 11.3, 28.2, 29.1; Th. 5.68.3; X. HG. 6.1.1, 4.14-15; Arist. Pol. 1270 a 16-17; Plu. Lyc. 8.5, 16.1 Hasta mediados de este siglo los autores se aventuraron a ofrecer algunas cifras sobre la población servil en Lacedemonia: K.J. BEL.OCH, Die Bevölkerung der Griechisch-Römischen Welt, Leipzig, 1889, 506 pensaba que con anterioridad a la Guerra del Peloponeso el número de hilotas rondaba los 175.000 , de los que 60.000 serían varones, alcanzando la población total de Laconia y Mesenia unos 230.000 habitantes aproximadamente; G. B. GRUNDY, "The Population and Policy of Sparta in the Fifth Century B.C.", JHS 28 (1908), 81 consideraba factible que la ratio de la población hilota respecto a la espartiata en el siglo $v$ fuera posiblemente de 1:15, es decir, 25.000 espartanos por 375.000 hilotas, cifra que no incluía a los periecos; actualmente, R.J. TALBERT, "The Role of the Helois in the Class Struggle at Sparta", Historia 38 (1989), 23 estima que el número aproximado de hilotas a mediados del siglo $v$ estaría entre 170.000-224.000, lo que coincidiria en líneas generales con las apreciaciones anteriormente expuestas. Cfr. también G.E.M. DE STE. Cholx, The Origins of the Peloponnesian War, Londres, 1972, 137-8 y 331-2 y T. J. FIGUEIRA, "Population Patterns in Late Archaic and Classical Sparta», TAPhA 116 (1986), 165-211.

11 Cfr. Plu. Lyc. 12.9 para el desarrollo de la ceremonia de admisión y Lyc. 12.8 para el silencio exigido a los miembros de la sussitia. 
de asociación política, en su significado más puro, servía de modelo a imitar para las hetairías y sinomosías de carácter oligárquico que en otros estados pretendían ser un grupo de presión social que intentaba imponer los intereses de los kaloikagathoi, es decir, de aquellos que por nacimiento, educación y modo de vida se consideraban los más aptos para gobernar a través de la Eunomia y la Eutaxia ${ }^{12}$. Respecto a la periodicidad de estas contribuciones a los banquetes, algunos autores han sostenido que eran mensuales ${ }^{13}$, si bien más que avalar tal hipótesis, las fuentes sugieren una mayor frecuencia de estas reuniones, celebradas siempre al anochecer ${ }^{14}$.

Como la mayor parte de las instituciones espartanas, el origen de la sussitia fue atribuido al mítico legislador Licurgo, con toda la problemática histórica y cronológica que presenta este personaje ${ }^{15}$. Jenofonte y Plutarco nos dicen que el legislador pretendía con ello fomentar el respeto y la obediencia a las leyes dictadas por el estado, al tiempo que reducir al mínimo la indisciplina reinante en la ciudad ${ }^{16}$. Aristóteles recoge también un deseo expresado por parte del legislador de introducir la comunidad de bienes en Esparta y Creta ${ }^{17}$. Al igual que gran parte de las instituciones, la que nos ocupa conservó su estructura y sentido original acuñado en época arcaica, sin mostrar transformaciones visibles hasta que con la Guerra del Peloponeso se inició una lenta evolución hacia formas de integración social que terminarán

12 Para la organización, fines y actividades de las hetairías, con un carácter general, puede consultarse G. M. CALHOUN, Athenian Clubs in Politics and Litigation, Austin, 1913, F. SARTORI, L'etairie nella vita politica ateniense del $V I$ e $V$ secolo a. C., Roma, 1957 y O. AURENCHE, Les groupes d'Alcibiade, de Leógoras et de Teneros. Remarques sur la vie politique athénienne en 415 av. J. C., París, 1974. En cuanto a la naturaleza e ideología de los grupos oligárquicos, véase $p$. ej. W. Donlan, The Aristocratic Ideal in Ancient Greece, Lawrence (Kansas), 1980 y L. WHEBLEY, Greek Oligrarchies. Their Character and Organisation, Chicago, $1975^{2}$.

${ }_{13}$ J. T. Hooker, op. cit., 134; P. A. Cartledge, op. cit,, 170; T. J. Figueira (1984), 89, n. ${ }^{\circ} 6$.

14 Plu. Lyc. 15.6. N.E.R. FISHER, op. cit., 32 las hace diarias sin argumentar su postura.

15 Hot. 1.65.4-5, X. Lac. 5.2 y Plut. Lyc. 12.1. Aristóteles (Pol. 1271 a 31-34, 1272 a 9 ) la atribuye «al legislador» de forma ambigua, que bien podría ser el propio Licurgo. A favor de la existencia histórica de este personaje se muestran, N.G.L. Hammond, "The Lycurgan Reform at Sparta", JHS 70 (1950), 57; G.L. HuXLEY, Early Sparta, Londres, 1962,7 y $42 ;$ W. G. FORREST, «Legislation in Sparta», Phoenix 17 (1963), 16-7. Se muestran dudosos, A. ANDREWES, The Greek Tyrants, Londres, 1956, 76; E. N. TIGERSTEDT, The Leyend of Sparta I, Estocolmo, 1965, 73. La niegan, al considerarlo un personaje mítico cuya existencia se encuentra vinculada a aspectos de índole ideológico, H. W. WADE-GERY, «The Growth of the Dorian State», CAH IH, Cambridge, 1925, 562 y V. EHRENBERG, Neugründer des Staates, Munich, 1925,30 y 49.

${ }_{16} \quad$ X. Lac. 5.2; Plut. Lyc. 8.2-3.

17 Arist. Pol. 1264 a 15. Cfr. también el posicionamiento adoptado por M. NAFIssI, op. cit., 191-206, al compararlo con la andreia practicada en Creta. 
por romper el anquilosamiento y tradicionalismo de la sociedad lacedemonia ${ }^{18}$. No obstante, a través de diversos textos literarios se puede observar que algunas instituciones lacedemarias habían roto parcialmente ese conservadurismo desde cuando menos el siglo VI; este es el caso de distintas formas de transmisión de la propiedad y del papel desempeñado por la mujer en el ámbito social espartano que, incluso, les diferenciaba de las estructuras apreciadas en el resto del mundo griego ${ }^{19}$. De esta forma, la sussitia simbolizó a la perfección hasta mediados del siglo III a. C., al menos teóricamente, las raíces de Esparta como estado conservador, totalitario y militarista; Plutarco recoge esta idea al decir que las comidas en común «reunían a los magistrados de las estirpes griegas que tienen las mejores Constituciones y conservan con más amor sus antiguas costumbres» ${ }^{20}$.

La dieta prescrita para estos banquetes se caracterizaba, según Polibio, por su frugalidad, con la intención de «hacer moderados a los hombres en sus vidas privadas y preservar de turbulencias la vida pública» ${ }^{21}$, algo que, como hemos expuesto con anterioridad, fue un objetivo primordial del legislador, a pesar de que otras fuentes literarias y arqueológicas parecen desmentir esta teórica limitación del alimento. Más fiable resulta la opinión de Jenofonte por su trato directo con las instituciones

18 Sobre las modificaciones producidas en Esparta a partir del siglo $\vee$ a. C. puede verse, G. Bockisch, «Harmostai», Klio 46 (1965), 150 ss.; P. A. CARTLEDGE, op. cit, 270 ss.; T. J. FIgueIRA (1984), 98; CH. HAMILTON, Agesilaus and the Failure of Spartan Hegemony, Itaca-Londres, 1991, 7085; E. BADIAN, «Agis II!", Hermes 95 (1967), 170-92. Para las reformas propuestas por Agis IV en el siglo III a. C., véase E. GabBA, "Studi su Filarco. Le biografie plutarche di Agide e Cleomene», Athenaeum 35 (1957), 13; A. FUKS, "The Spartan Citizen-body in Mid-third Century B. C. and its Enlargement Proposed by Agis IV ", Athenaeum 40 (1962), 244-63; E. DAVID, Sparta between Empire and Revolution (404-243 B.C.), Nueva York, 1981, 174, n. ${ }^{\circ} 11$; B. SHIMRON, Late Sparta: the Spartan Revolution, 243-146 B.C., Búfalo, 1972; J. R. F. MARTínEZ-LACY, «Agis's and Cleomenes's Reforms and their Relationship to Expressions and Appearances of Resistence by the Ruled", en T. YUGE-M. Dol (eds.), Forms of Control and Subordination in Antiquity, Leiden, 1988, 476-81.

19 Véase, a título de ejemplo, Hdt. 5.39-41; 6.57.4-5; 6.71; x. HG 5.3.9; Mem. 1.2.61; Plu. Cim. 10.6 .

20 Plu. Mor. $714 \mathrm{~B}$. Aunque no podemos descartar que algunas instituciones espartanas se remonten a la época oscura, la mayoría, y entre ellas la sussitia, parecen datar de principios del siglo VII, en conexión con la conquista de Mesenia y el subsiguiente aumento de la tierra productiva distribuida entre los espartiatas: cfr. P. A. CARTLEDGE, op. cit., 170. Sobre el gran valor económico de estas nuevas tierras puede verse, J. KroYmann, Sparta und Messenien. Untersuchungen zur überlieferung der messenischen Kriege, Berlín, 1937, 14; H. MicHEL, op. cit., 206, y J. M. CASILLAS y C. FORNIS, «Resonancias épicas en Tirteo: Aproximación al contenido histórico de los poemas 4D y 6 + 7D», en III Coloquio de Estudiantes de Filologia Clásica: Poesía épica griega y latina (Valdepeñas, 1991), Valdepeñas, 1992, 208, 210-1 y 215.

21 PIb. 6.48.3. Se trata en definitiva, como apunta N.R.E. FISHER, op. cit., 30, de evitar las manifestaciones de hybris. 
lacedemonias en plena época clásica, quien en diferentes pasajes de su República de los Lacedemonios considera que estas comidas eran suficientes para calmar el apetito sin caer en excesos ${ }^{22}$. Además, esta moderación se manifestaba mayormente en la falta de consumo de productos exóticos y no en la escasez de alimentos, que los espartiatas ingerían por encima de la media del soldado griego ${ }^{23}$. Este hecho se enmarca convenientemente en el carácter y educación recibida por los espartiatas, cuya temprana dedicación a la vida militar exigía un cuerpo ágil a la vez que poderoso para la disposición y entrega bélica. En cualquier caso, se trataba de cantidades nada despreciables de alimentos en relación a la producción media de los kleroi ${ }^{24}$.

Por otra parte, a diferencia de la mayoría de los sumposia griegos, en las sussitiai los simposiastas no acababan ebrios, haciendo honor a la fama de moderados bebedores que tenían los espartanos, característica que se extendía a todo tipo de fiestas y celebraciones ${ }^{25}$. Sin duda, como

22 X. Lac. 2.5-6; 5.3. De esta manera, se pretendía conseguir en el espartiata un cuerpo esbelto que, complementado con el diario entrenamiento físico y militar, permitiera desarrollar adecuadamente la masa muscular.

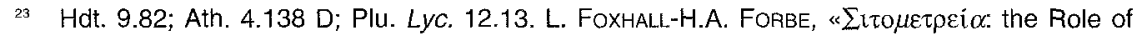
Grain as a Staple Food in Classical Antiquity", Chiron 12 (1982), 59 ha precisado el valor calórico de la comida aportada a la sussitia en $4.230 \mathrm{kcal}$. por día y hombre, superando entre 408 y $893 \mathrm{kcal}$. lo requerido por los más activos varones adultos. Cfr. lo expuesto por T. J. Figueira (1984), $92-94$ para un minucioso estudio de los hábitos alimenticios y productos más consumidos por los espartanos.

${ }^{24}$ T. J. FIGUEIRA (1984), 98-9 ha estimado la aportación a la sussitia en aproximadamente un $37 \%$ de la producción mensual del kleros.

25 Para el vino en la sussitia, cfr. Plu. Lyc. 12.14; 15.6; para las Huacinthas, Ath. 4.139 D; véase tambiên el texto de X. Lac. 5.4-7 y entre los estudios modernos, M. NAFISsI, op. cit., 178-91, B. C. DiETRICH, «The Dorian Hyacinthia: a Survival from the Bronze Age», Kadmos 14 (1975), 13342, M. E. IRWIN, “Odysseus 'Hyacinthine hair' in Odyssey 6.231», Phoenix 44 (1990), 205-18, L. y F. VILlARD, «Hyakinthos», LIMC 5 (1991), 546-50, M. PETtersson, Cults of Apollo at Sparta. The Hyakinthia, the Gymnopaidiai and the Karnea, Estocolmo, 1992, 9-41 y L. BruIT, «The meal at the Hyakinthia: ritual consumption and offering", en O. MurraY (ed.), Sympotica: a Symposium on the Symposion, Oxford, 1990, 162-74; para las Gimnopedias, B. D. MERRIT, «The Spartan Gymnopaidia", CPh 26 (1931), 70-84. En Esparta existía otra comida, el kolpis, relacionada sobre todo con el culto a Artemis, aunque en algunas ocasiones también se consumía durante las celebraciones a Apolo. No tenía la signił́icación exclusivista de la sussitia, pues en estas conmemoraciones se repartía a todos los participantes, incluidas mujeres y determinada población dependiente como los periecos, pero también podian comerlo los xenoi que estuviesen en la ciudad de Esparta durante estas destacadas fiestas religiosas. Por tanto, se disolvían, aunque fuese momentáneamente, las diversas categorías diferenciadas de la sociedad espartana: hombre-mujer, adulto-adolescente, ciudadano-dependiente y lacedemonio-extranjero. La comida se componia de

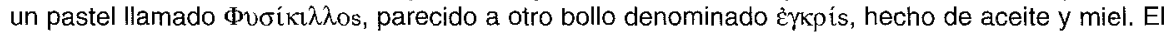

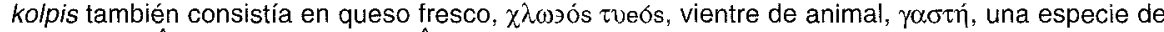

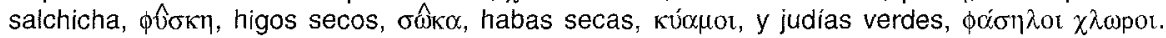
Véase, Ath. 4.138 E-F; 139 A-B y Hdt. 9.82. También puede consultarse los trabajos de W. KROLL, «Kolpis», RE IX, Stuttgart, 1922, 1362-3; F. BÖLTE, «Zu lakonischen Festen», RhM 78 (1929), 134-35; H. JeAnMaiRe, Couroi et Courètes. Essai sur l'éducation spartiate et sur les rites d'adolescence dans l'antiquité hellénique, Travaux et mémoires de l'Université de Lille 21, Lille, 1939, 524- 
dice Plutarco, los homoioi no querían «hacer menos útiles los banquetes viriles" ${ }^{26}$, enturbiando el buen desarrollo y significado de los mismos.

En cambio, quienes sí bebían vino en gran cantidad eran los hilotas, hasta el punto de cantar y bailar de forma ridícula, lo que en opinión de T. J. Figueira constituía «un refuerzo psicológico de su inferioridad ante los espartiatas" ${ }^{27}$. Este autor toma como base la supuesta presencia de hilotas sirviendo las mesas y la elevada consumición de comida y bebida para argumentar que la sussitia "no sólo era una institución para la vida comunal de los espartiatas, sino un mecanismo de redistribución del alimento según la jerarquía social» ${ }^{28}$. Sin negar la función económica de este banquete, que proveía para las clases desfavorecidas un complemento a su alimentación habitual, consideramos, no obstante, que Figueira va más allá de lo permisible en el aspecto puramente social al postular que hilotas y mujeres solteras participaban de estas celebraciones ${ }^{29}$. Mientras que el propio autor admite su falta de base para explicar la presencia de mujeres solteras, resulta inadmisible pensar que la población servil tuviera acceso a conversaciones, ritos, en algunos casos iniciáticos, y otro tipo de manifestaciones de la elite sociopolítica cuando a los propios espartiatas se les obligaba a guardar silencio y cuando, por otra parte, los hilotas eran un peligro potencial endémico para la estabilidad del estado lacedemonio ${ }^{30}$. Por contra, debemos ajustarnos a la información de las fuentes, que en

40; J. M. VERPOORTEN, "La 'stibas' ou l'image de la brousse dans la société grecque», RHR 162 (1962), 147-60; W. BuRKERT, Greek Religion. Archaic and Classical, Oxford, 1985, 107; U. KRON, «Kultmahle im Heraion von Samos archaischer Zeit. Versuch einer Rekonstrucktion», en R. HÄGGN. Marinatos-G. C. Nordquist (eds.), Early Greek Cult Practice, Estocolmo, 1988, 138.

${ }_{26}$ Plu. Mor. 218 D 4.

27 T. J. FIGUEIRA (1984), 97.

28 Ibid., seguido por N.R.E. FISHER, op. cit, 34. A su hipótesis, que ya fue apuntada por L. FOXHALL-H.A. FORBES, op. cit., 59 como una posibilidad alternativa a la utilización de los productos sobrantes para el comercio, contribuye sin duda el hecho de que se trata de productos perecederos $y$, por tanto, de imposible almacenamiento.

29 Ibiol.

30 Cir. Plu. Lyc. 28.8, con la introducción del rito de la crupteia a partir del terremoto de $c .464$ y Th. 4.80 para la eliminación de dos mil hilotas en el 424 ante una posible rebelión tras el de. sastre de Esfacteria. Los hilotas podian ser de procedencia laconia o mesenia; éstos últimos, mucho más numerosos según Th. 1.101.2, al tener conciencia étnica, siempre aprovecharon cualquier desastre natural o militar para reivindicar y luchar por su perdida libertad, sólo recuperada tras el reconocimiento del estado mesenio en 369 por Epaminondas (D.S. 15.66.1; 66.6; Plu. Ages. 34.1; Pelop. 24.9; Paus. 4.26.5-27). Sobre la mayor disposición a la revuelta de la servidumbre étnica con respecto a la esclavitud mercancia, véase $p$. ej. CL. Mossé, "Le rôle des es claves dans les troubles politiques du monde grec à la fin de l'époque classique", $C H 6,4$ (1961), 353-60 y G.E.M. DE STE. CROIX, La lucha de clases en el mundo griego antiguo (trad. de T. de Lozoya), Barcelona, 1988, 116, 336, 661-2; una visión diferente en D. M. LEWIS, «Ithome again», Historia 3 (1954/5), 412-8. Véase también lo expuesto por É. Lévy, «La Kryptie et ses contradictions», Ktema 13 (1988), 245-52. 
ningún momento presentan a los hilotas participando del banquete, sino que les atribuyen un papel de meros bufones, precisando además que la ingestión de alcohol se producía al margen de las mesas espartiatas y que sólo al final los hilotas eran introducidos en el sussition como ejemplo educacional para que los más jóvenes conocieran los efectos de la ebriedad en el hombre ${ }^{31}$.

Precisamente a los jóvenes se les permitía el acceso al sussition en calidad de auditores como complemento a su educación. En este sentido, en presencia de los jóvenes los adultos se comportaban respetuosamente, evitando bromas de mal gusto y enfados ${ }^{32}$. La agoge contemplaba que el joven fuera introducido en actividades y actos sociales como hetairías, gimnasios y banquetes, de acuerdo a los presupuestos de ocio productivo y digno (schole) reservados a los espartiatas ${ }^{33}$. Se establecía así un vínculo entre los adultos con plena capacidad política y los jóvenes destinados a aprender los mecanismos de poder que cristalizaba en la mayoría de los casos en una relación de tipo homosexual. Las asociaciones exclusivamente masculinas que ensalzaban las virtudes viriles fomentaban que el joven buscara entre sus integrantes un modelo a imitar, mientras el amante adulto elegido asumía la potestad moral de guía y conductor (relación erastes-eromenos), en el seno de una cultura donde el estado se hace cargo de la educación del individuo desde una temprana edad, aun a costa de romper los vínculos familiares o de sangre ${ }^{34}$. No en vano Jenofonte afirma con rotundidad que la pederastia era considerada $\tau \dot{\eta} \mathrm{v}$ $\kappa \alpha \lambda \hat{i} \mathrm{i} \sigma \tau \eta \nu \pi \alpha \delta_{\varepsilon i \alpha{ }^{35}}$.

Los textos más específicos acerca de los alimentos y dinero aportados por cada uno de los comensales a la sussitia corresponden a Plutarco y Dicearco de Mesenia, quienes hablan de las siguientes cantidades: un

\footnotetext{
${ }_{31}$ Plu. Lyc. 28.8-9; Demetr. 1.5; Mor. 239 A. Nuestra opinión es compartida por M. NAFISSI, op. cit., 190; sin embargo, J. DuCAT, "Le mépris des hilotes", Annales (ESC) 29 (1974), 1457-8 no cree que ésta sea la finalidad del ritual, sino más bien poner de manifiesto el menosprecio hacia los hilotas.

${ }_{32}$ Plu. Lyc. 12.6; X. Lac. 5.6. En la sussitia se mantenía una rígida jerarquia por clases de edad en las que los jóvenes prestaban obediencia a los mayores (cfr. al respecto N.R.E. FISHER, op. cit., 37).

${ }_{33}$ H. I. Marrou, Histoire de l'éducation dans l'Antiquité, París, 1948, 67; A. BILlHeIMER, «AgeClasses in Sparta in Education», TAPhA 78 (1947), 99-104.

${ }_{34}$ Plu. Lyc. 18; Arist. Pol. 1272 a 9; Ar. Lys. 79-84; cfr. H. I. MARRou, op. cit., 66, K. J. Dover, Greek Homosexuality, Londres, 1978, 193, B. L. KUnstLER, op. cit., 435 y P. A. CARTLEDGE, "The Politics of Spartan Pederasty", PCPhS n.s. 27 (1981), 17-36. Pl. Lg. 8.836 C hace a los gimnasios responsables del origen de la pederastia en Creta y Lacedemonia.
}

${ }_{35}$ X. LaC. 2.13. 
medimno de cebada (74 kg.), ocho choes de vino (el choe laconio equivale a 4,62 lt.), cinco minas de queso (mina $=437$ gr.), cinco semiminas de higos y una pequeña contribución en dinero, que Dicearco precisa en diez óbolos eginetas, para provisiones extra ${ }^{36}$. Sin embargo, es probable que Plutarco se refiera a una aportación media del grupo, más que a una forma individualizada, pues no de todos los $\kappa \lambda \hat{\eta} \rho$ or se obtenía una homogeneidad cuantitativa y cualitativa en los productos, sino que la contribución dependía de su situación geográfica, del tipo de cultivo y de la fertilidad de la tierra ${ }^{37}$. Así por ejemplo, había ciudadanos con un especial grado de riqueza que se permitían el lujo de contribuir a la mesa con pan de trigo ${ }^{38}$.

Pero la desigualdad en las aportaciones al banquete se hace más patente en el postre ( $\varepsilon \pi \alpha \hat{i} \kappa \lambda o v)$, de carácter voluntario y servido al tiempo que se anunciaba, por parte del $\mu$ óyctpos, el nombre del donante, hecho en

${ }^{36}$ Plu. Lyc. 12.3. Dicearco (fr. 72 Wehrli = Ath. $4.141 \mathrm{C}$ ) nos transmite la misma lista de alimentos, pero traducidos, en cambio, a medidas áticas: medimno y medio de cebada $(51,84 \mathrm{~kg}$.), ocho choes de vino (3,24 lt. el choe), queso e higos sin especificar el peso concreto y los ya mencionados óbolos eginetas. Por otra parte, T. J. FigueIRA (1984), 89 considera que los espartiatas, en lugar de dinero, aportarían comida por valor de esos diez óbolos, que se toman como patrón económico por estar más familiarizados con la moneda egineta, común entre sus aliados peloponesios. Las monedas espartanas más antiguas, fechadas en la primera mitad del siglo III, son tetradracmas de plata que imitan las emisiones de Alejandro Magno y que llevan el nombre del rey Areo, que reinó entre el 309 y el 264 a. C. (Plu. Plys. 17.2-3; X. Lac. 7.5); véase, B. V. HEAD, Historia Numerum, Oxford, 1911, 434; C. T. SELTMAN, Greek Coins, Londres, 1955, 256. Con posterioridad, en el año 228 el monarca Cleómenes lli acuñó tetradracmas de plata y después también lo hizo Nabis, en ambos casos imitando las emisiones de los Seléucidas; véase el planteamiento arqueológico de H. MiCHEL, op. cit., 302 y el más conservador de M. I. RostovtzeFf, Historia económica y social del mundo helenistico, Madrid, 1967, 186.

${ }_{36}$ Este doble desequilibrio, cuantitativo y cualitativo, en la posesión de la tierra ya sería constatable desde el temprano arcaísmo, apareciendo reflejado en un texto de Aristóteles $(P$ ol. 1270 a 13-14), que haría mención más a un tiempo pretérito que a su propia época [J. J. KEANEY, «Hignett's HAC and the Autorship of the Athenaion Politeia", LCM 5 (1980), 5-6]. Por tanto habría espartiatas que disfrutarían de importantes parcelas de tierra, mientras que otras tendrían que conformarse con un minifundio. Esta diferenciación vendría marcada desde la propia situación geográfica en que se encontraba emplazado el dominio territorial de cada una de las familias espartiatas. Las mejores tierras estaban situadas en el valle del río Eurotas y en la zona oeste de Mesenia (según P. A. CARTLEDGE, Agesilaos and the Crisis of Sparta, Londres-Baltimore, 1987, 173 unas 50.000-75.000 Has., que se ubicaban muy probablemente en un área cercana a la ciudad de Esparta y a la llanura del Helos, adecuadas por su feracidad para la creación de cuatro mil kleroi de entre 11 y 18 Has.). Estas cifras, sobre todo la primera, coinciden con las establecidas en 1961 por el censo realizado por el gobierno heleno, que daba una extensión de $47.153 \mathrm{Has}$. de suelo cultivable, en las que habría que incluir tanto tierras excelentes para la agricultura como otras situadas en un terreno más montañoso, de mayor dificultad y menor calidad para el cultivo [el resto del territorio estaría distribuido entre comunidades de origen perieco; G. SHIPLEY, «Perioikos: The Discovery of Classical Lakonia", en J. MOTIKA SANDERS (ed.), Laconian Studies in honour of Hector Catling, Atenas, 1992, 211-26]. Los kleroi espartiatas estarían enfocados a una producción de subsistencia al no existir un especial incentivo para la venta local o la exportación, ni tampoco un deseo de mantener los excedentes agrícolas, pues la población ciudadana no se ocupaba en actividades banáusicas.

${ }^{37}$ Alcm. fr. 95 a Page; X. Lac. 5.3. 
el que hemos de ver una indudable finalidad de ganar prestigio social ante los sussitoi, en la competencia constante (agon) que caracterizaba el ethos aristocrático en cualquier comunidad ${ }^{39}$. El epaiklon promovía igualmente una diferenciación por clases de edad ya que existían dos tipos según quien lo consumía: uno servido a los adolescentes, paides ${ }^{40}$, todavía no integrados plenamente en la comunidad ciudadana, el otro reservado para el resto de los miembros ${ }^{41}$. De esta manera, mejor que pensar con N.R.E. Fisher que los compañeros de mesa menos favorecidos económicamente agradecían esta práctica evergética concebida como manifestación solidaria que reafirmaba la cohesión del cuerpo social ${ }^{42}$, se nos antoja más plausible que bajo esta aparente uniformidad emanada de la sussitia subyace una realidad menos ideal, salpicada de las lógicas tensiones generadas por las luchas de poder entre los diferentes círculos políticos que, al modo de las hetaireiai, se agrupaban en torno a un personaje destacado y pugnaban por imponer sus tesis ${ }^{43}$.

${ }_{38}$ Ath. 4.141 D. Cfr. L. FoxhALl.-H. A. Forbes, op. cit., 58-9 y T. J. FIGUeIRA (1984), 90. El componente competitivo se encuentra siempre asociado a todo tipo de simposio (O. MURRAY, op. cit., passim).

39 Consistía en un bollo de cebada empapado en aceite, que se engullía ávidamente con hojas de laurel (Ath. 4.140 D-E).

${ }^{40}$ Bollo compuesto de carne procedente bien de la caza obtenida por los propios espartiatas, bien de los rebaños que éstos poseían (Ath. 4.141 D-E). Posiblemente esta elaboración marque por sí misma una cierta diferenciación entre los propios espartiatas si pensamos que no todos serían propietarios de ganado y, por tanto, se demostraba así una mayor disponibilidad de medios.

${ }^{41}$ Op. cit, 37-9. De forma similar M. PeTterson, op. cit., 17 sostiene que el epaiklon podía ayudar a que el joven se acomodara progresivamente en el grupo, creando así un espíritu de solidaridad que sólo se logra en las comunidades humanas con el reparto de comida en la mesa; cfr. también L. BRUIT, op. cit., 171-2.

42 Para un acercamiento a estos conflictos internos, una de cuyas manifestaciones más evidentes era la desigual y contradictoria política exterior en momentos especialmente críticos de la historia espartana, véase S. HODKINSON, "Social Order and the Conflict of Values in Classical Sparta", Chiron 13 (1983), 239-81; id., "Land Tenure and Inheritance in Classical Sparta», CQ n.s. 36 (1986), 378-406; P. A. CARTLEDGE (1987), 203-41; M. I. FinleY, «Sparta», en J. P. VernaNT (ed.), Problèmes de la guerre en Grèce ancienne, París, 1968, 143-60; G. E. M. DE STE CRoIx, op. cit., 151-61; P. A. BRUNT, «Spartan Policy and Strategy in Archidamian War», Phoenix 19 (1965), 255-80; E. BARH-HEN, «Le parti de la paix à la veille de la Guerre du Peloponnèse», AncSoc 8 (1977), 21-31; TH. KELLY, "Thucydides and Spartan Strategy in the Archidamian War», AHR 87 (1983), 25-54; I. MoxON, «Thucydides' Account of Spartan Strategy and Foreign Policy in the Archidamian War», RSA 8 (1978), 7-26; J. T. HoOkER, «Spartan Propaganda», en A. PowELL (ed.), op. cit., 122-4i; A. GRIFFITHS, "Was Kleomenes Mad?», en ibid., 551-78; D. HARVEY, «Leonidas the Regicide? Speculations on the Death of Kleomenes I", en Arktowros. Hellenistic Studies to B.M.W. Knox, Berlín, 1979, 253-60; E.F. BloEDOw, «The Speeches of Archidamos and Sthenelaidas at Sparta», Historia 30 (1981), 125-43; U. BULTRIGHINI, "I| "pacifismo" di Archidamo: Tucidide e i suoi interpreti», RCCM 33,1 (1991), 5-28; D.G. RICE, Why Sparta Failed: a Study of Politics and Policy from the Peace of Antalcidas to the Battle of Leuctra, 387-371 B.C., diss. Yale University 1971, passim; CH. HAMILTON, op. cit, passim; E. DAVID (1981), passim. 
La desigualdad en la producción de los kleroi y el afán competitivo demostrado en la comida en común nos conduce a la problemática de si realmente los homoioi eran tales. En una opinión claramente consecuente con lo expuesto, no, al menos en el plano práctico ${ }^{44}$. Un espartiata, en principio, tenía una especial consideración social por pertenecer a la elite étnica, consideración que podía verse notablemente incrementada a través del desempeño de una magistratura o de un reconocimiento que revirtiese en el prestigio de la ciudad, sea en combate o en unos juegos panhelénicos ${ }^{45}$. Un claro signo de diferenciación social era la crianza y entrenamiento de caballos, en calidad de hippotrophoi, privilegio y excelencia de los aristoi, que hacian así ostentación de su linaje y patrimonio; Esparta no permaneció ajena a esta práctica, según demuestra la predilección de algunos homoioi por presentar carros a competición ${ }^{46}$. Como veremos más adelante, la Guerra del Peloponeso supuso una notable ampliación de las diferencias sociales.

${ }_{43}$ Así, es probable que dentro de los homoioi tuvieran especial consideración los trescientos Hippeis y los Agathoergoi encargados de las misiones secretas (Hdt. 1.67). Cfr. J. P. VERNANT \& P. VIDAL-NAQUET, Travail et esclavage en Grèce Ancienne, Bruselas, 1989, 87; en contra, J. F. LAZENBY, The Spartan Army, Warminster, 1985, 53. Estamos de acuerdo con P. A. CARTLEde, «Hoplites and Heroes», JHS 97 (1977), 27 cuando dice: «if we can give a concrete sense to the self-styled Spartan homoioi (peers), it is to uniformity of their hoplite equipment and training, not to the equally of their nor even their shared way of life as a whole, that we should primarily loof»; del mismo modo, P. VIDAL-

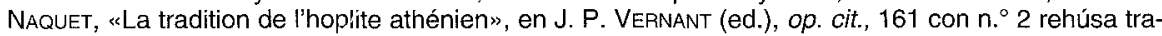
ducir el término hornoioi por «iguales" y prefiere darle la connotación de «semejantes"; cfr. también id., op. cit., 161-2. Igualmente, debemos tener en cuenta que por muy igualitaria que pretenda ser una sociedad, dando entrada a los mecanismos de gobierno a todo el cuerpo cívico, determinados individuos no estarán nunca conformes con este reparto teórico y ambicionarán más poder personal de acuerdo a unos méritos que creen encarnar; cfr. A. G. WOODHEAD, “Conflict and Ancient Society», en J. W. Aluison (ed.), Conflict, Antithesis and the Ancient Historian, Columbo, 1990, 9-10. Por consiguiente, en Esparta esa igualdad no existía en la práctica, sino que se veía alterada por la existencia de la doble realeza, dotada de un origen mítico: los reyes eran personajes privilegiados cuya posición social se debía en la mayoría de los casos a su nacimiento (a su pertenencia a la familia Agiada o Euripóntida) y no a unos méritos personales intrínsecos; a su lado resulta evidente la existencia de una aristocracia dentro del grupo de «iguales", acaparadora de una mayor riqueza - de acuerdo a la producción de sus kleroi-e influencia -campeones olímpicos o héroes ciudadanosy, por consiguiente, por encima del resto de sus homónimos ciudadanos. La evolución de la Esparta clásica tendió a reforzar esas diferencias sociales y a favorecer la concentración de riqueza entre un número más o menos limitado de ciudadanos de pleno derecho; cfr. M. AUSTIN \& P. VIDAL-NAQUET, op. cit., 86. FINLEY, op. cit., 146-52 remarca especialmente que la oposición entre individuos ambiciosos y de fuerte personalidad y el resto de la ciudadanía era fuente continua de tensión interna y concluye que los «iguales», al fin y al cabo, se veían inmersos en un complejo de desigualdades.

${ }_{44}$ Ejemplo de esto podemos encontrarlos en Samos, donde se halló la tumba de Arquias, enviado a la isla por Esparta en misión diplomática (Plu. Mor. 601 B; Pi. 0. 6.28), en Atenas, las diversas sepulturas de los soldados lacedemonios caídos en 403 (G. KARO, An Attic Cemetery, Filadelfia, 1943, 25-6) o los santuarios dedicados a Marón y Alfeo, los primeros espartanos muertos en las Termópilas (Paus. 3.12.9); finalmente, Evágoras, vencedor con sus yeguas en tres olimpiadas consecutivas a fines del siglo VI (Hdt. 6.103.4) y Damanes y su hijo, cuyas victorias olímpicas fueron perpetuadas en una inscripción colocada a finales del siglo $v$ en el santuario de Atenea en la Acrópolis de Esparta [S. HoDKinson (1983), 260].

${ }_{45}$ Isoc. 6.55; Paus. 6.2.1. Efectivamente, durante el período comprendido entre c. 548 y $c$. 
Según la legislación atribuida a Licurgo, en la sociedad espartiata el estado controlaba la administración de los kleroi a través de los homoioi, siendo el beneficiario último de la propiedad a la muerte de su posesor si la tierra no tenía un heredero a quien le correspondiese. No obstante, el reparto inalienable de la tierra en Esparta es un mito forjado a mediados del siglo iv ${ }^{47}$ ya que las evidencias que tenemos parecen sugerir que Éforo atribuyó la idea de la redistribución igualitaria de la propiedad a Licurgo ${ }^{48}$. Lo que posiblemente podría permanecer inalterable hasta finales del siglo $v$ sería la indivisibilidad de la tierra, ya que ésta, al pasar a un único heredero, el hijo mayor, queda en manos de un solo posesor que la retiene en su poder hasta su muerte ${ }^{49}$. Así, podríamos proponer la existencia de un sistema de propiedad fundamentalmente privado e indivisible hasta comienzos del siglo IV que admitiera la transmisión de los bienes inmuebles a través de la herencia, mientras los valores mobiliarios serían susceptibles de ser cedidos en vida de su dueño mediante donación, legados testamentarios o esponsales de los herederos ${ }^{50}$. La permisividad constitucional producto de una mayor apertura hacia el exterior tras la Guerra del Peloponeso posibilitó la acumulación de tierras en

368 se produjeron numerosas victorias olímpicas espartanas en la prueba de carrera de carros (Pausanias asegura que ningún otro estado griego lo superó en este apartado). Véase M. A. FLOWER, «Revolutionary Agitation and Social Change in Classical Sparta», en M. A. FLOWER-M. TOHER (eds.), Georgica. Greek Studies in Honour of George Cawkwell, Londres, 1991, 89-90 y G.E.M. DE STE. CrolX, sp. cit., Apénd. XXVIII, que citan numerosos pasajes como ejemplo. Para una lista completa de vencedores olímpicos en esta modalidad de carrera, se puede consultar L. MORETTI, Olympionikai, i vincitori negli antichi agoni, Atti della Academia Nazionale dei Lincei, Classe di Scienze Morali, Storia e Filologia, Ser. 8, 8, 2, Roma, 1959, 58 ss., completada en id., «Supplemento al catalogi degli Olympionikai», Klio 52 (1970), 295 ss. Una discusión completa sobre este particular puede encontrarse en S. HODKINSON, «Inheritance, Marriage and Demography: Perspectives upon the Success and Decline of Classical Sparta», en A. PowELL (ed.), op. cit., 97, en donde se llega a algunas conclusiones interesantes. Sobre la importancia social del caballo en la vida cultural espartana, cfr. también Alcm. fr. 7 Page. En cuanto a su presencia en la arqueología se puede consultar P. M. FRASER, «Archaeology in Greece: Sparta», $A R 17(1970 / 1)$, 14. Igualmente no es de extrañar que durante época helenística se pudiesen enviar caballos desde Mesenia a Alejandría; cfr. Plu. Cleom. 35.3 y C. RoEBUCK, "A Note on Messenian Economy and Population", CPh 40 (1945), 155.

${ }_{46} \mathrm{Plb}, 6.45 .3$, que cita en este texto a Éforo, Platón, Jenofonte y Calístenes, es decir, todos autores del siglo Iv. Cfr. A.H.M. JONES, op. cit., 43; S. HODKINSON (1986), 381-4; M. A. FLOWER, op. cit., 81-2.

47 Ephor. FGH 70 F 118. Cfr. F.W. WALBANK, A Historical Commentary on Polybius I, Oxford, 1957, 728; G.L. BARBER, The Historian Ephorus, Cambridge, 1935, 116; específicamente sobre la visión idealista que Polibio tenia de Esparta, véase E. LEVY, «La Sparte de Polybe», Ktema 12 (1987), 63-79.

48 P.A. CARTLEDGE, «Spartan Wife: Liberation or Licence?», CQ n.s. 31 (1981), 99-102; S. HODKINSON (1986), 394-404. Contra D. ASHERl, "Land of Inheritance. Distribution of Land and Political Constitution in Ancient Greece», Historia 12 (1963), 5 y P. Otiva, Esparta y sus problemas sociales (trad. de M. Picazo), Madrid, 1983, 39, que considera que el lote del padre pasaba normalemente a su hijo e incluso a más de un hijo o hija a la vez, con lo que la propiedad podría ser compartida por varios hermanos, para lo que se basa en un texto de Plu. Agis 5.1 .

${ }_{49}$ Arist. Pol. 1270 a 13-14; Heraclid. Lemb. fr. 12 Dilts; Plu. Mor. 238 E. Para un comentario más amplio sobre estos textos, véase M.R. DiLts, Heraclidis Lembi Excerpta Politiarum, GRBS monografia 5 (1971), passim. 
manos de unos pocos gene cuyos miembros habían fundamentado la base de su riqueza en los beneficios obtenidos mediante el ejercicio de un cargo, ya fuese político, militar o administrativo, fuera de Laconia ${ }^{51}$, empobreciendo al mismo tiempo a gran número de ciudadanos que pasaron a integrar una masa poblacional que permanentemente podía contribuir a la inestabilidad de la ciudad.

Considerando este panorama sociopolítico, podemos fácilmente imaginar lo que suponía para un homoios, un espartiata de nacimiento, la pérdida de sus derechos políticos. A esta terrible consecuencia se podía llegar, entre otros casos, si no se aportaba a la sussitia los productos en las cantidades estipuladas. De esta manera, el espartiata era despojado de su condición de «semejante» y relegado a una ciudadanía de segunda clase ${ }^{52}$. Son los llamados $v \pi$ o $\mu$ cioves, carentes de derechos políticos, que no civiles, pues el kleros era un derecho consustancial a su condición de espartiata ${ }^{53}$. Su lugar en la sociedad no debía de ser muy diferente del ocupado por los llamados tresantes, aquellos que habían sido degradados de su estatus de homoios por cobardía o desobediencia en el desarrollo de una campaña militar ${ }^{54}$, al de aquellos que habían fracasado en algún estadio de la agoge o habían sido encausados bajo los delitos de atimia o asebeia ${ }^{55}$. Todos ellos se encontraban situados en el orden social entre los neodamodes - esclavos liberados - y los periecos - semidependientes encargados de las labores y trabajos manuales y del comercio-, si bien hemos de suponer que un hupomeion no sufriese la humillación y

51 Esto se encuentra atestiguado en la totalidad de las fuentes (X. Lac. 7.6, 14, An. 2.6.1-5; Eph. FGH 70 F 148-9; Plu. Lyc. 30.1, Agis 4.1, 5.1, 5.5, Lys. 17, Mor. 239 D-240 A; Arist. fr. 544 Rose; Posidonius FGH 87 F 48; D.S. 14.12; Ael. VH. 14.29; Theompomp. FGH 115 F 192 y 232...). Por ejemplo, Gilipo, navarca espartano — conductor de la flota lacedemonia en Sicilia durante la Guerra del Peloponeso-, fue acusado de quedarse con dinero para su uso y enriquecimiento personal, por lo que fue desterrado a comienzos del siglo IV. Véase A.J. TOYNBEE, «THE GROWTH OF SPARTA», JHS 33 (1913), 272; M. SARY, "Notes on the History of the Fourth Century», CQ 20 (1926), 186; id., «The Ascendancy of Sparta», CAH VI, Cambridge, 1927, 29; E. DAVID (1981), 6-8; CH. HAMILTON, Sparta's Bitter Victories: Politics and Diplomacy in the Corinthian War, Itaca, 1979, 55-8; R. VATTUONE, "La congiura di Cinadone», RSA 12 (1982), 22-7.

52 Arist. Pol. 1271 a 31,1272 a 7 ; cf. X. Lac. 10.7.

53 El estatuto de hupomeion no está atestiguado antes de la revuelta de Cinadón en 398 (X. HG. 3.3.6; 3.11), aunque los prisioneros de Esfacteria sufrieron una degradación política a su regreso a Esparta en 421/0 (Th. 5.34.2), que tal vez significara de facto el paso a una condición de hupomeion. Cfr. Y. GarlaN, Slavery in Ancient Greece (trad. de J. Lloyd), Londres, 1988, 98; CH. HaMil.ton (1991), 70 y T.J. Figueira (1986), 196.

54 Hdt. 7.23; Plu. Lyc. 21.2; Ages. 30.3; X. Lac. 9.4-6. Cfr. N. LorauX, «La "belle morte" spartiate", Ktema 2 (1977), 108-12; J.P. VernANT \& P. VIDAL-NAQUET, op. cit., 87 y 112 ; M.A. FLOWER, op. cit., 84.

${ }_{55}$ M.I. FINLEY, op. cit., 153. 
muestras de repulsa dirigidas a impíos, malversadores de bienes públicos - cobardes, ya que, después de todo, la causa de su desgracia no era otra cosa que la penuria económica. El ejemplo más representativo de hupomeion fue Cinadón, quien en el 398 a. C. lideró una conspiración integrada por las clases dependientes contra los homoioi ${ }^{56}$. Esta marginación producto de no cumplir con los requerimientos del banquete es criticada por Aristóteles, quien juzgaba más equitativa y justa la organización de la comida en común cretense, donde todos los gastos corrían a cargo del erario público y es en esta apreciación donde funda el Estagirita la esencia de la ciudadanía ${ }^{57}$.

Otro aspecto que debemos considerar es la posible participación de los $\mu$ ó $\theta \alpha \kappa \varepsilon$ s en la comida en común espartana. Este término en griego hacia referencia a los hijos de los espartiatas que habían perdido el estatus de homoios, es decir, de aquellos que por su aptitud habian traicionado los ideales de Licurgo ${ }^{58}$, lo que les privaba de la plena ciudadanía. No obstante, los mothakes estaban estrechamente unidos ideológicamente a la elite política y militar lacedemonia y mantenian una alta posición social en virtud de su nacimiento y educación, común a todos los espartiatas. Además, ellos podían alcanzar la plena ciudadanía mediante la realización de algún servicio distinguido al Estado. Desde comienzos del siglo IV podían también acumular una considerable riqueza, como fue el caso de Lisandro, que, de acuerdo a Plutarco, antes de alcanzar una posición próxima a la de homoios y con ello la plena ciudadanía, tuvo que realizar destacados servicios militares durante la Guerra Jónica ${ }^{59}$; asimismo,

${ }_{56}$ X. HG. 3.3.4-11; Arist. Pol. 1306 b 3; Max. Tyr. 35.8 C; Polyaen. 2.14.1. Artículos especificos sobre la figura de Cinadón son los de R. VATTUONE, op. cit, 19-52 y E. DAVID, «The Conspiracy of Cinadon», Athenaeum 57 (1979); cfr. también P.A. CARTLedge (1979), 273-5, 321-4, CL. Mossé, op. cit., 355-6, P. Oliva, op. cit, 170, 195-6, G.L. CAKWELl, «The Decline of Sparta», CQ n.s. 33 (1983), 391, M.A. FlowER, op. cit., 94-5. Una segunda conspiración contra el sistema, que tal vez contara con la participación de hupomeiones y otros inferiores - los hupouloi kai poneroi de Plutarco-, fue la desarrollada en $370 / 69$, poco después de la derrota de Leuctra, con los tebanos a las puertas del Peloponeso (Plu. Ages. 32.5; Nep. 6.2-3; Polyaen. 2.1.14).

57 Arist. Pol. 1271 a 32; de esta forma, en Esparta la sussitia pierde el factor integrador que pretendía el legislador (Arist. Pol. 1264 a 15), reemplazado por cierta marginación sociopolítica.

58 Phylarch. FGH 81 F 43; Ael. VH. 12.43; Th. 6.93.2; Plu. Nic. 28.5; cfr. P.A. CARTLEdGE (1987), 28. En contra de esta tesis se muestran K.M. CHRIMES, op. cit., 97, 117 y 220-3, F.R. Wüst, «Laconia", Klio 37 (1959), 60-3, H. MiCHEL, op. cit., 89, que defienden el estatuto servil de los mothakes. Otra posibilidad es la mantenida por D. LOZTZE, «Mothakes», Historia 11 (1962), 427, P. Oliva, op. cit., 176-8, Y. Garlan, op. cit, 98, W.G. Forrest (1980), 136, M. Austin y P. VIDAL-NAQUET, op. cit., 91-2, y M. NAFISSI, op. cit., 175 n. 74, que engloban bajo este epígrafe no solamente a los hijos de los homoioi privados de la plena ciudadanía, sino también a los hijos bastardos fruto de uniones mixtas entre espartiatas e inferiores.

59 Plu. Lys. 1.3, 2.2; Ael. VH. 12.43. Cfr. Ath. 6.27 E-F. 
tenemos los ejemplos de Calicrátides y Gilipo, que obtuvieron la ciudadanía a fines de la Guerra del Peloponeso ${ }^{60}$. Todos ellos eran mothakes, desempeñaron el cargo de navarca y, con posterioridad, llegaron a la plena ciudadanía y adquirieron un gran poder personal. Por tanto, no puede extrañarnos que un $\mu$ ó $\theta \alpha \xi$ tomase parte a título individual en la mesa común y asimilase los aspectos ideológicos de los homoioi en la sussitia ${ }^{61}$.

En este contexto social es inevitable hacer referencia a la ausencia de mujeres en las sussitiai cuando su presencia en la vida cívica espartana era importante en diversos ámbitos (económico, intelectual, deportivo, etc.), aunque no en la toma de decisiones políticas ${ }^{62}$. La razón hemos de encontrarla en la profunda separación de sexos existente en Esparta, que exigía a los hombres una mutua convivencia desde los siete años en todos los órdenes, lo que propició la carencia de relaciones afectivas con las mujeres hasta el momento de contraer matrimonio; incluso entonces, la mujer necesitaba ser vestida de forma varonil para que en la oscuridad el hombre no sufriese un impacto psicológico ante un acto al que la agoge no le había acostumbrado, abandonando el lecho conyugal al poco tiempo para ir en busca de sus compañeros ${ }^{63}$. Por tanto, la dedicación del espartiata varón a la Ekklesia, al ejército, a las asociaciones, etc., le inhibía de las relaciones hombre-mujer en favor de la homosexualidad. La sussitia no fue una excepción debido a su profundo significado político, social y militar exclusivo de los óv $\delta \rho \varepsilon \zeta$, lo que explica la ausencia en la misma de todo tipo de mujeres, incluidas las hetairai, habituales en la mayoría de los banquetes griegos.

50 Sobre la personalidad de Gilipo, véase Plu. Lys. 16-17.1 y Ael. VH. 12.43; 14.19. Este personaje era hijo de un distinguido espartiata, Cleándridas, que fue exiliado en 445 bajo la acusación de soborno y, por consiguiente, privado de sus derechos de ciudadanía (Plu. Per. 22.3 y Nic. 28.5). Para Calicrátides, Ael. VH. 12.43. Cfr. P.A. Cartledge (1979), 28.

\$1 En general, G.L. CAWKWELL, op. cit., 394.

62 Sobre la importancia de la mujer en Esparta, véase PI. Lg. 806 A; Arist. Pol. 1269 b 5-7; Plu. Lyc. 14.1-2; Theoc. 18-22. Para un planteamiento radical sobre el tema del control económico por parte de las mismas, se debe consultar lo expuesto por B.L. KunsTLer, op. cit., 423. Para el papel general de las mujeres en la sociedad lacedemonia, P.A. CARTLEdGE (1981), 84-105. Así, las mujeres casadas, junto a madres, hermanas e hijas, participaban activamente en las ceremonias fúnebres de monarcas; cfr. J.M. CASILLAS, "Geras Thanontôn: Muerte y funerales en la monarquía lacedemonia", Polis 5 (1993), 37.

63 Plu. Lyc. 15.5; 15.7. Así se explicarian dos hechos diferenciadores respecto de la media griega: por una parte, varones y mujeres contraían matrimonio a una edad más tardia (Plu. Lyc. 15.4) y, por otra, el alto porcentaje de mujeres que llegaban vírgenes a las nupcias, lo que probaría la escasez de relaciones heterosexuales durante el periodo educacional del varón, a pesar de la permisividad existente con el adulterio; cfr. P.A. CARTLEDGE (1983), 91 y K.J. Dover, op. cit., 193. 
La asistencia al sussition era obligatoria para todos los espartiatas y sólo se excusaba si uno se encontraba cazando o realizando un sacrificio, en cuyo caso debían enviarse a la mesa común parte de las capturas obtenidas en la caza o las primicias del sacrificio ${ }^{64}$. Un derecho potestativo del monarca lacedemonio, de los pocos que en la práctica mantenía, era poder participar de la sussitia sin asistir al sussition, de modo que en su hogar recibia dos quénives de harina de cebada y un cótilo de vino; si decidía acudir a la comida en común, se le ofrecía doble ración de todos los alimentos ${ }^{65}$. Sin embargo, este derecho real debía de estar sujeto a algún tipo de control por parte de los magistrados de la polis, ya que por ejemplo a Agis II le fue prohibida la celebración en privado, con su esposa, de una victoria sobre los atenienses en la Guerra del Peloponeso; cuando envió a recoger su ración y ésta le fue negada por los polemarcos, su indignación le motivó incluso la imposición de una multa al rehusar hacer un sacrificio ${ }^{66}$.

El hecho de que el polemarcado sea un cargo militar apunta a un posible origen de la sussitia en el marco de las campañas exteriores, en cuyo desarrollo la comida en común (óyóvıov) constituía una parte fundamental de la convivencia diaria de los espartanos, permitiendo crear, entre otros aspectos, lazos de unión y camaradería que hacian tomar conciencia de la necesaria protección mutua entre los compañeros de mesa. En este caso, los comensales de la misma eran los enomotes, es decir, los integrantes de la unidad inferior del ejército espartano denominada enomotia ${ }^{67}$. En este sentido la edad mínima para integrarse de una forma definitiva en las comidas en común estaba en torno a los veinte años, aproximadamente la misma que era necesaria para formar parte del ejército ${ }^{68}$. No es necesario recordar que la guerra para el estado espartano era una prolongación de la vida ciudadana ya que sólo podían tomar las armas los espartiatas, mientras que la población dependiente lo hacía únicamente en casos de extrema necesidad. Heródoto también parece aludir a este origen en el seno

${ }_{64}$ Plu. Lyc. 12.4; X. Lac. 5.3.

65 Hdt. 6.57.3; según T.J. FIGUEIRA (1984), 97 los reyes recibian mayor cantidad de comida por tener un número mayor de hilotas a su servicio, de acuerdo a su argumentación de redistribución de alimentos entre la población servil. Por otra parte, N.R.E. FiSHER, op. cit., 32 con n. 33, siguiendo a P. CARLIER, La royauté en Grèce avant Alexandre, Estrasburgo, 1984, 250 ss., arguye que posiblemente estos privilegios reales fueran ya obsoletos en tiempos de Heródoto.

66 Plu. Lyc. 12.5.

67 Tanto W.G. ForREST (1980), 46 como J.F. LAZENBY, op. cit., 7 y 12 consideran que la enomotia se componía de cuarenta espartiatas.

es Cfr. P.A. Cartledge (1981), 22 y N.R.E. Fisher, op. cit., 33, W.G. Forrest (1980), 52 eleva la edad mínima a los treinta años, poniéndola en relación con el acceso a las sesiones de la Ekklesia. 
del ejército al incluir la sussitia entre los reglamentos militares, junto a las enomatiai y las triecades ${ }^{69}$.

Como último punto que cierre nuestro estudio vamos a centrarnos en los cambios experimentados por la sussitia a mediados del siglo III a. C., encuadrados en las renovaciones constitucionales efectuadas por Agis IV. Desde finales del siglo $v$ se produjo en Esparta una progresiva reducción del derecho a la ciudadanía como consecuencia de una mayor diferenciación social en el grado de riqueza monetaria y fundiaria, producto a su vez del mantenimiento del imperio heredado de la Guerra del Peloponeso ${ }^{70}$, a la vez que una creciente disminución de la población espartiata. Las cifras en este sentido son indicativas: en 418 habría unos tres mil seiscientos espartiatas varones ${ }^{71}$, antes del desastre de Leuctra (371) la cifra se había reducido a 1.200-1.000 ${ }^{72}$, a mediados del siglo IV eran ya menos de un millar ${ }^{73} \mathrm{y}$, por último, a mediados del siglo ill no superaban los setecientos ${ }^{74}$. Las motivaciones de este hecho son diversas y variadas, sin que debiéramos encontrarlas exclusivamente en las razones moralizantes expuestas por Plutarco ${ }^{75}$ y por algunos autores contemporáneos ${ }^{76}$, que se basan fundamentalmente en el influjo que el dinero causó en Esparta a comienzos del siglo IV. En esta creciente oliganthropia también tuvieron una importancia destacada otros aspectos, como la desigualdad de la propiedad entre los ciudadanos y la penetración constante de población dependiente en el ejército lacedemonio ${ }^{77}$. Mientras unos pocos homoioi, que

99 Hdt. 1.65.5.

70 Como hemos avanzado arriba (vid. n. 50), el desempeño de cargos políticos y militares en el imperio abrió un abismo dentro de la población espartiata, donde se consolidó una aristocracia ciudadana minoritaria frente al crecimiento numérico de las clases inferiores, no acostumbradas a los mecanismo del librecambio; entre éstas se encontraban los hupomeiones, quienes, como hemos mencionado anteriormente, por la pérdida de la propiedad veían suprimidos sus derechos políticos y la posibilidad de formar parte de la elite militar y social lacedemonia. Cfr. G.L. CAWKWELL, op. cit., 387-90 y E. DAVID (1981), 46-50; contra, W.G. ForREST (1980), 124-5, A.J. HolladAy, «Spartan Austerity», $C Q$ n.s. 27 (1977), 118-21, M.A. Flower, op. cit., 88-94 y S. HODKINSON (1986), 386-94.

71 Th. 5.68.3.

72 X. HG. 6.4.14-15; cf. 6.1.1.

73 Arist. Pol. 1270 a $16-17$.

74 Plut. Agis 5.5.

${ }_{75}$ Plu. Lys. 17; Mor. 239 F. Cfr. Plu. Lys. 30 y Arist. fr. 501 Rose. Sobre el afán moralizante del de Queronea, véase S. HODKINSON (1986), 382-3; C.G. STARR, «The Credibility of Early Spartan History", Historia 14 (1965), 257-72; M.A. FLOWER, op. cit., 81-3.

${ }_{76}$ E. DAVID, "The Influx of Money into Sparta at the End of the Fifth Century B.C.", SCI 5 (1979/80), 30-45, esp. 38; cfr. también G.L. CAWKWELL, op. cit., 395-7.

77 Se pueden citar diversos y constantes ejemplos sobre este hecho. Ya durante la batalla de Platea en 479 participaron, junto al ejército espartano compuesto de homoioi, cinco mil periecos formando una unidad independiente y treinta y cinco mil hilotas que acompañaron a sus respectivos 
coinciden con las familias que se reparten el poder político, se enriquecían notablemente, el resto sufría un empobrecimiento e incluso la necesidad de vender el kleros, lo que en muchas ocasiones suponía la incapacidad para pagar los costes de la sussitia y, por ende, la inmediata pérdida de los derechos políticos ${ }^{78}$.

Consecuentemente la disminución en número de ciudadanos varones revirtió en un aumento del porcentaje de tierra cultivable en posesión de mujeres, un $40 \%$ a mediados del siglo IV, a la que accedían ahora a través de la herencia, y en la posibilidad de que los hupomeiones buscaran en el matrimonio con estas propietarias un medio de acceso a la ciudadanía perdida ${ }^{79}$. Todo ello redundó en una continuada debilidad del estado espartano en época helenística.

Dentro de este planteamiento hemos de entender las reformas institucionales de Agis IV (244-241 a. C.), entre las cuales se incluye la concesión de

amos (Hdt. 9.10.1; 11.3; 28.2; cfr. 7.103.3). Con posterioridad, esta participación se incrementó, pero fue fundamentalmente a partir de mediados de la Guerra del Peloponeso cuando su concurso aumentó de una manera significativa, coincidiendo con la disminución del número de ciudadanos. Así, en 421 surge por primera vez el nombre de neodamodeis, es decir, "el nuevo damos". En 413 los espartanos junto a seiscientos hoplitas hilotas y neodamodes marchan a Sicilia, donde adquirieron experiencia en la guerra (Th. 6.19.3); durante la Guerra Jónica un comandante perieco mandó una escuadra de treinta naves (Th. 8.22.1); asimismo, el segundo comandante de la expedición espartana para ayudar a Ciro en su revolución, Neón de Asine, era posiblemente un perieco (X. An. 1.4.3; 5.3.4). Este fenómeno se prolongó durante el siglo IV: Agesilao fue acompañado por tres mil neodamodes en la expedición a Asia y en 382 contó en el ejército con un importante número de hupomeiones y periecos (X. HG. $6.2 .5 ; 4.9$ ), al igual que ła utilización, por necesidad, de periecos durante la campaña de Agesípolis en Olinto (X. HG. 5.3.9, cfr. D.S. 15.22.2). Véase E. CAVAIGNAC, "A propos de la bataille du torrent de Némée (Juin 394)", REA 27 (1925), 275-8; W.K. PRITCHETT, Studies in Greek Topography II, Berkeley-Los Ángeles, 1969, 75-6; J.K. ANDERSon, Military Practice in the Age of Xenophon, Berkeley-Los Ángeles, 1970, 226-7; P.A. CARTLEdge (1979), 280-1; J.F. LaZenby, op. cit., 17-20; P. Oliva, op. cit., 179 y T.J. Figueira (1986), 200-5; más específicamente, T. ALFIERI TONINI, " "ll problema dei neodamodeis nell'ambito della società spartana", RIL 109 (1975), 305-16; M. FUROYAMA, "The Liberation of Heilotai. The Case of Neodamodeis", en T. YUGE-M. Dol (eds.), op. cit., 364-8; R.F. WILLETS, "The neodamodeis", CPh 49 (1954), 27-32; G.B. BrunI, "Mothakes, neodamodeis, brasideoi", en Schiavitù, manomissioni e classi dipendenti nel mondo antico, Roma, 1979, 21-31.

${ }_{78}$ El progresivo incremento en número y malestar de las clases sociales inferiores tuvo una primera expresión en la mencionada revuelta de Cinadón (vid. supra n. 55). Por otro lado, el hieratismo de la legislación de Licurgo impedía a los ciudadanos dedicarse a actividades onerosas como el artesanado o el comercio, habitualmente en manos de periecos, limitando así las perspectivas de futuro de los ciudadanos de segunda clase (X. Lac. 7.1-2); cfr. P.A. CARTLEDGE, «Did Spartan Citizens ever Practice a Manual Tekhne?", LCM 1 (1976), 115-9. y G. BerTHIAUME, "Citoyens spécialistes à Sparte», Mnemosyne 29, 4 (1975), 360-4.

79 En Esparta la mujer no heredaba a menos que no hubiese varones en la familia, pero desde el siglo iv tenemos constancia de que la escasez de ciudadanos posibilitó que las mujeres pudieran recibir al menos parte de la herencia (Arist. Pol. 1270 a 14-15). Un buen estudio sobre este papel de la mujer en el régimen de propiedad y en la coyuntura demográfica de la Esparta del siglo iv es el de S. HODKINSON (1989), 82-9; cfr. también B.L. KUNSTLER, op. cit., 481 y 484 y supra n. ${ }^{\circ} 61$. 
derechos políticos a una parte importante de la población no ciudadana ${ }^{80}$. Dichos cambios trajeron consigo, a su vez, la vuelta de algunas tradiciones de la agoge espartana, que desde hacía más de un siglo había caído en desuso ${ }^{81}$ y que fueron impuestas tanto a los hijos de los antiguos como de los nuevos ciudadanos con la finalidad de recuperar el viejo espíritu del mítico legislador Licurgo. También esto significó, entre otras cosas, una regulación en el régimen de las sussitiai, de la que sólo tenemos constancia por la variación en la distribución de los comensales, que pasan de estar agrupados en mesas de quince personas en época clásica ${ }^{82}$ a repartirse en grupos de entre doscientos y cuatrocientos individuos por cada una de las quince mesas establecidas ${ }^{83}$, posiblemente con el objetivo de que sirviesen de integración a los nuevos ciudadanos. Desgraciadamente, no sabemos si esta modificación se extendió a otros aspectos de la originaria organización de las comidas en común ${ }^{84}$, pero, se limitara o no esta reforma al ámbito puramente numérico, lo que sí resulta evidente es que, con la integración de nuevos ciudadanos, la sussitia alteró su primigenia significación de reforzamiento e identificación de la elite sociopolítica espartiata ${ }^{85}$.

3o Plu. Agis 8.2; cfr. J.F. LAZENBY, op. cit., 182; G. BOCKISCH, op. cit, 150; A. FuKS, op. cit., P.A. Cartledge (1979), 270 ss.; T.J. Figueira (1984), 98; CH. Hamilton (1991), 70-85; E. GabBa, op. cit., 13 y E. DAVID (1981), 124 n. 11. Además, el rey Agis IV procedió a la cancelación total de deudas de la población de Laconia, pero se le impidió continuar con la redistribución de tierras que pretendía realizar (cfr. Plu. Cleom. 17.5 y B. SHIMRON, op. cit., 9-26).

${ }^{81}$ Cfr. M.A. FLOWER, op. cit., 79.

82 Plu. Lyc. 12.2.

в3 Plu. Agis 8.3. Cfr. P.A. Cartledge-A. Spawforth, Hellenistic and Roman Sparta. A Tale of Two Cities, Londres, 1992, 41, 46 y 52; L.J. PIPERS, "Spartan Helots in the Hellenistic Age», AS 15-17 (1984/5), 75-88.

${ }_{84}$ W.G. FORREST (1980), 45 y J.F. LAZENBY, op. cit., 182 defienden que la reforma se restringió al número de componentes y no afectó a otros aspectos de la institución; por contra, T.J. FIGUEIRA (1984), 98 con n. 32 sitúa la reforma de la sussitia entre el 265-254 (por tanto fuera del reinado de Agis IV), dejando en todo caso de ser un mecanismo integrador de la población dependiente para convertirse en un medio de ayuda del espartiata rico al pobre.

85. Como consecuencia de las radicales transformaciones efectuadas por el rey Nabis (206192 a. C.), se dio entrada en todas las instituciones, incluida la sussitia, a la población dependiente (cfr. P.A. CARTledge-A. SPaWforth, op. cit., 78). A pesar de que con la conquista romana la $\mathrm{CO}-$ mida en común acentúa su decadencia, bajo Domiciano, a finales del siglo I d. C. y posteriormente por los emperadores filohelenos del siglo $\|$, el simbolismo de esta institución todavía perdura hasta el punto de aglutinar en torno a sí a la aristocracia espartana romanizada (P.A. CARTLEDGEA. SPAWFORTH, 106, 199-200). 